\title{
The role of heart rate variability in sports physiology (Review)
}

\author{
JIN-GUO DONG \\ Institute of Physical Education, Chifeng University, Chifeng, Inner Mongolia 024000, P.R. China
}

Received December 1, 2015; Accepted February 23, 2016

DOI: $10.3892 /$ etm.2016.3104

\begin{abstract}
Heart rate variability (HRV) is a relevant marker reflecting cardiac modulation by sympathetic and vagal components of the autonomic nervous system (ANS). Although the clinical application of HRV is mainly associated with the prediction of sudden cardiac death and assessing cardiovascular and metabolic illness progression, recent observations have suggested its applicability to physical exercise training. HRV is becoming one of the most useful tools for tracking the time course of training adaptation/maladaptation of athletes and in setting the optimal training loads leading to improved performances. However, little is known regarding the role of HRV and the internal effects of physical exercise on an athlete, which may be useful in designing fitness programs ensuring sufficient training load that may correspond with the specific ability of the athlete. In this review, we offer a comprehensive assessment of investigations concerning the interrelation between HRV and ANS, and examine how the application of HRV to physical exercise may play a role in sports physiology.
\end{abstract}

\section{Contents}

1. Introduction

2. Heart rate variability (HRV)

3. HRV and sports physiology

4. Conclusion

\section{Introduction}

The heart is a specialised pump that functions by regular and continuous contractions for delivery of blood throughout the body (1). The pumping action is caused by a flow of electricity through the heart that repeats itself in a cycle, known as heart rate (HR) or heart pulse. HR is the speed of the heartbeat measured by the number of contractions per unit of time (2),

Correspondence to: Jin-Guo Dong, Institute of Physical Education, Chifeng University, 1 Yingbin Road, Chifeng, Inner Mongolia 024000, P.R. China

E-mail: v281098@163.com

Key words: heart rate, heart rate variability, sport, autonomic nervous system a measure determined by calculating the heart rate variability (HRV) from electrocardiogram (ECG) recordings.

The understanding of the significance of HRV is ongoing. However, it has been suggested that HRV is an important method for assessing cardiovascular autonomic parameters that are partially under the regulatory control of innervations from the sympathetic and parasympathetic systems $(3,4)$. Notably, these two components of the autonomic nervous system (ANS) balance between them affects the consistency in the time between heart beats. Consequently, HRV reflects oscillations in the heart cycle duration over time and is generally considered the measure of regulatory influences, mainly of the activity of ANS to regulate the function of the cardiovascular system. Previous findings have identified the potential use of HRV for recognizing healthy and diseased states since the vagal-mediated HRV indices were inversely associated with several risk factors for diabetes, glucose intolerance, insulin resistance, central obesity, dyslipidemia and hypertension (3).

However, while HRV was largely applied to predict sudden cardiac death and diabetic neuropathies in assessing disease progression $(5,6)$, recent studies demonstrated the application of HRV in exercise training. Their findings supported the use of HRV as a marker to reflect the cardiac modulation of the sympathetic and vagal component of the ANS (7), and suggested that monitoring indices of HRV may be useful for tracking the time course of training adaptation/maladaptation in order to set optimal training loads that lead to improved performances (3,7-9). Specifically, the aim of all training is the use of enough physical training loads to the body to transfer homeostasis and the whole autonomic balance. Athletes may better adapt their physiology during a sufficient recovery of the body (10). However, how long- and short-term effects of training and the condition of athletes, as well as the efficiency of the training, and the recovery level to maximize the benefits of training remain to be determined.

Nevertheless, studies $(2,11,12)$ comparing ANS between sedentary and active subjects or athletes of different sports modalities have shown different HRV profiles, suggesting the possibility of monitoring HRV indices for improving physical and physiological states.

The present review examined the possible role of HRV in sports physiology. Particularly, we examined the autonomic regulation of the heart and its relationship with HRV, as well as the relevance of the use of HRV in sport training with consideration of the intensity of the training, the level of athletes, the gender and age of athletes and, the use of HRV to monitor and improve sports physiology. 


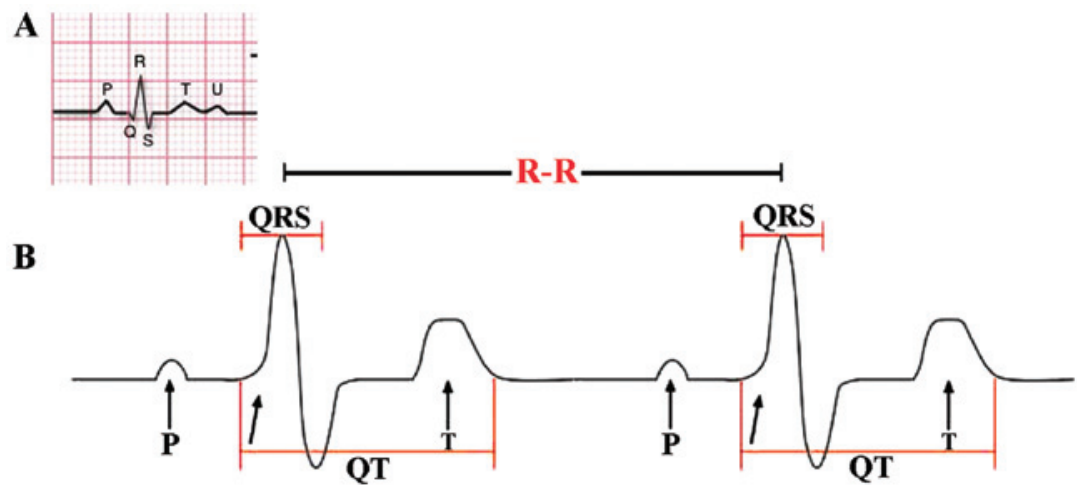

Figure 1. Electrocardiogram (ECG) as the basis of measuring heart rate variability (HRV). (A) The electrical activity of the heart is detected by ECG. The waveform components indicate an electrical event during one heart beat. The waveforms are labeled P (first short upward movement of the ECG tracing), the QRS complex (Q-larger upwards deflexion, R-a larger upwards deflexion, S-downwards wave) shows ventricular depolarization and contraction. PR indicates the transit time required for the electrical signal to travel from the sinus to the ventricles of the heart. The T-wave is normally a modest waveform representing ventricular repolarisation. (B) Representation of HRV monitored and analysed to determine the state of the nervous system controlling the heart. The ANS is determined by detecting variation in consecutive time intervals between peaks of the QRS complex, R-R interval (red). Originally, HRV was measured manually from calculation of the mean R-R interval and its deviation was measured on short-term ECGs. Lower HRV corresponds to the smaller standard deviation in R-R intervals. There are several types of arithmetic manipulation of R-R intervals to represent HVR $(34,62)$.

\section{Heart rate variability $(\mathrm{HRV})$}

Autonomic innervations of the heart. The heart and circulatory system are primarily controlled by the higher brain center (central command) and by the cardiovascular control area located in the brain stem, through the activity of the ANS. The ANS comprises the sympathetic and parasympathetic nerves (vagal nerves) outflow to the heart and blood vessels, which are primarily regulated by the medulla (13). Particularly, the nucleus tractus solitarius in the medulla receives sensory input and stimulates cardiovascular responses for emotion and physical stress. From the medulla, the parasympathetic vagus nerve innervates the heart to the sympathetic nerve fibers. The right and left vagus nerves innervate the sinus atrial (SA) and atrioventricular nodes, respectively. The atria are also innervated by vagal efferent, whereas the ventricular myocardium is sparsely innervated by the vagal efferent. Sympathetic efferent nerves are present throughout the atria, particularly in the SA node and ventricles. Sympathetic stimulation increases the HR, and contractility and conduction velocity through the mediation of $\alpha$ and $\beta$ adrenoreceptors. Parasympathetic stimulation has the opposite effects through the muscarinic receptor. Autonomic control of the cardiovascular system is also affected by baroreceptors, chemoreceptor, muscle afferents, local tissue metabolism, circulating hormones, and environmental behaviour (14-16 as well as ethnic group $(17,18)$. Although sympathetic and parasympathetic systems are active at rest, the parasympathetic fibers release acetylcholine, which acts to retard the pacemaker's potential of the SA node and thus reduce the HR (19).

ANS regulation of HRV. HRV refers to the beat-to-beat alteration of the heart. The ECG of a healthy individual measured under resting conditions shows periodic variation consisting of a rhythmic phenomenon known as respiratory sinus arrhythmia (RSA). RSA fluctuates with the phase of respiration with cardio-acceleration during inspiration and cardio-deceleration during expiration. Vagal efferent trafficking to the sinus node occurred primarily in the phase with expiration, and absent or attenuated during inspiration. These data identify RSA as predominantly mediated by respiratory gating of parasympathetic efferent activity to the heart; referring HRV as a marker of dynamic and cumulative loads. As a dynamic marker of loads, HRV appears to be sensitive and responsive to acute stress. Experimentally, it has been shown that mental load (i.e., making complex decisions, public speaking tasks) decrease HRV. As a marker of cumulative wear and tear, HRV is also reduced with the aging process. Significant resting HR does not change with aging (20) and a reduction of HRV was attributed to a decrease in efferent vagal tone and reduced $\beta$-adrenergic responsiveness. By contrast, regular physical activity retards the aging process, increasing HRV, presumably by increasing vagal tone. Therefore, HRV is considered a marker of frequent activation (short dips in HRV in response to acute stress) and the inadequate response (long-term vagal withdrawal, resulting in the over-activity of the counter-regulatory system), leading to the sympathetic control of cardiac rhythm.

Basic measurement of HRV parameters and interpretations. HRV may be evaluated by a variety of complex methods. The most common method is standard ECG, considering the temporal variation between the sequences of consecutive heart beats (Fig. 1). The lengths of successive R peaks (R-R) in the QRS complex can be described mathematically. R-R is not consistent between successive $\mathrm{R}$ peaks. Of note, during the onset of physical activity, R-R intervals become shorter and more uniform, resulting from increased sympathetic activity and parasympathetic withdrawal. R-R can provide actionable information regarding the physiological stress and fatigue levels during and after training (21). Thus, despite the complexity of the type of mathematics involved in the calculation of HRV, a variety of algorithmic models that represent R-R intervals are widely available, and autonomic activation can be evaluated by analysing HRV to estimate the sympathetic-vagal balance $(22,23)$. In addition, the period between the QRS complex resulting from sinus node depolarisations is termed the normal-normal $(\mathrm{N}-\mathrm{N})$ interval. $\mathrm{HRV}$ is the measurement of the variability of the N-N intervals (24). Additionally, frequency-domain analysis describes high and low frequency (LV) rates of the variability changes, 
corresponding to the activity of different branches of ANS. By applying these frequency range differences in HRV analysis, the individual contribution of parasympathetic and sympathetic systems were identified. Parameters LF referring to modulation of the R-R interval changes between 0.04 and $0.15 \mathrm{~Hz}$ corresponds to the sympathetic and parasympathetic activity together. High frequency (HF) modulation $(0.15-0.4 \mathrm{~Hz})$ of R-R interval changes is primarily regulated through innervations of the heart by the parasympathetic (vagal) nerve (25). LF and HF parameters are provided as normalized (LFn and HFn) by calculating the fraction of $\mathrm{LF}$ or $\mathrm{HF}$ relative to the total minus LF (26). Another crucial element for the analysis of HRV is the time-domain parameters reflecting the standard deviation (SD) of all N-N intervals (SDNN) that reproduce the total variability and the root mean square of SDs between adjacent N-N intervals (RMSSD), which reflect parasympathetic activity $(2,16,27)$. Another parameter that may be considered is the Pointcaré plot, calculated as follows: an individual's R-R intervals plotted over time and SD used to interpret changes are evident in the plot (28). The standard descriptor 1 (SD1) is the fast beat-to-beat variability in the R-R intervals, while the standard descriptor 2 (SD2) describes the longer-term variability (29). SD1 reflects mainly the parasympathetic input to the heart, while SD2 reflects the sympathetic and parasympathetic contributions to the heart.

However, as mentioned earlier, respiration greatly affects HRV, thereby increasing HRV when respiratory frequency decreases, rendering difficult the proper interpretation of HRV data. Thus, investigators have accepted various respiratory frequency ranges (i.e., 6-15 beats/min) and admitted self-organized respiratory pattern to be maintained during the recording period $(17,23)$, in order to have interpretable results.

\section{HRV and sports physiology}

Application of HRV on sports training. Although there are notable physical and physiological differences between athletes training for different sporting activities (15), HRV is becoming one of the most used training and recovery monitoring tools in sport sciences $(31,32)$. The possibility of applying HRV on such variety is based on the fact that cardiovascular autonomic regulation is an important determinant of training adaptations, before also being responsive to training effects (33). In concordance with these observations, an ANS comparison between sedentary subjects and recreationally active subjects or athletes of different sports modalities have demonstrated that athletes exhibit a different HRV profile to sedentary control subjects, with an overall increase in HRV and parasympathetic cardiac modulation (12), while evidence suggests that high-intensity training can chronically lead to a shift vagal to sympathetic cardiac modulation. In recreational marathon athletes, the progressive sympathetic predominance at peak training load may predict performances in the race (13). Thus, a transition from vagal to sympathetic predominance in cardiovascular autonomic modulation, ranging from low intensities to peak training loads on world-class rowers has been reported $(2,34,35)$. It has also been shown that endurance and team athletic activities induce an elevated parasympathetic modulation over the $24 \mathrm{~h}$ recording period (higher RMSDD, PNN50 and HF, and lower LF/HF ratio) (36). Thus, endurance elite athletes have an elevated parasympathetic tone compared to recreational athletes and non-athletes; confirming that athletic conditioning is an important variable that influences the autonomic control of the heart.

Application of HRV on the intensity of sports training. The HR varies relative to the body's physical needs, such as the need to absorb oxygen and excrete carbon dioxide, physical exercise, sleep, anxiety, stress, illness, ingesting and drugs (37-39). In athletes, the sympathovagal balance is altered in response to different intensities and duration of aerobic training. This was evidenced by changes in HRV variables measured, including LF, HF and the total power of frequency domain, in an intensity-dependent manner (13). At baseline, the LFn was slightly greater than that of the HFn. The ratio LF/HF increased over time and reflected the sympathetic tone (increased LFnu) and the decrease in the parasympathetic tone (decreased HFnu) (13). The results of HRV spectra indicated by LFn and HFn in exercise vary in function to ventilatory thresholds (VTs) $(40,41)$ and identified a sympathetic predominance (indicated by an increased $\mathrm{LF} / \mathrm{HF}$ ratio) during relatively less intense exercise and parasympathetic predominance (indicated by a decreased $\mathrm{LF} / \mathrm{HF}$ ratio) during relatively more intense exercise.

Investigation of regional elite triathletes $(\sim 15$ years old) challenged with moderate-intensity test (below VT power) or high-intensity test (above VT power) until exhaustion, Cottin et al (40) measured the R-R interval together with VO2, VCO2 (42) and blood lactate levels.

The authors found higher absolute LF, HF, and TP values at moderate-intensity compared to high-intensity cycling. Normalization showed LFn was significantly higher in cycling below VT, indicating predominance of the sympathetic input while the opposite was observed during heavy exercise, indicating a predominance of the parasympathetic input (40). These data may be explained by a change in the breathing rate combined with disappearance of the cardiac autonomic control that occurs with heavy exercise conditions. Furthermore the $\mathrm{LF} / \mathrm{HF}$ ratio was always $>1$ for cycling below $\mathrm{VT}$ and $<1$ for cycling above VT (40). This gradual switch ratio suggests the overstepping of the VT and presents be a reliable index of VT detection from the HRV.

Application of HRV on the effects of gender and age on training. The beneficial effects of physical exercise on enhancing vagal tone have been identified (43). Using SD1 normalized (SD1n) for average R-R intervals on subjects from different age groups and conditioning showed that SD1n was significantly higher at rest in the young subjects (24-34 years old) compared with the middle-aged (35-46 years) or elderly (47-64 years). These age-related differences in cardiac vagal activity were not significant after exercise (44). The age-matched subjects with good, average and poor VO2 peaks showed no difference in SD1n at rest, but were significantly different in the low- to moderate-intensity levels. These findings suggest that poor physical fitness was associated with impairment in cardiac vagal function during physical exercise. Enhanced vagal activity in youth was more evident at rest (45). However, endurance athletes have a higher parasympathetic tone than sedentary subjects and the effects of resistance training on athletes showed gender differences resulting from training (45). Indeed, significant differences in selected 
domains between genders, including NFnm, LFnm, LH, LHnu and PNN50 indicated that the autonomic control of the heart is not merely a question of conditioning (45). Thus aging, gender and athletic conditioning are important variables that influence the autonomic control of the heart in athletes.

Application of HRV on the effects of sports training. Scientific data have shown that HRV taken immediately after exercise during recovery reflects characteristic responses indicating the body is to exercise, which is correlated with athletic fitness (46). Accordingly, an analysis of HRV identified that HFn, SD1, SD2 and SDNN were decreased on the first day after maximal exercise compared to the pre-exercise value in 10 healthy male cross-country skiers of $\sim 36$ years of age during a $75-\mathrm{km}$ race. On the second day, HFn returned to the baseline level, with LFn been reduced to near or below pre-exercise levels on the second day after the race, revealing a reduction in the parasympathetic tone after a cross-country skiing performance and demonstrating recovery time inversely correlated with the VO2 max measured during a bicycle test prior to the race. The reduced vagal outflow appeared to be blunted after prolonged exercise dependent on the cardiorespiratory fitness of the athlete. An accentuated rebound of altered regulation was found on the second day after prolonged exercise. By linking HRV measurements to VO2 max, the intensity of the exercise (VO2 max) was correlated with the HRV measurements. In addition, HRV indices derived from the traditional time-consuming methods have been shown to be sensitive effects in team sports players $(11,47,48)$, using single measure per period of assessment. Averaging multiple measures per week to increase the confidence of data and sensitivity to detect changes associated with variations in training loads $(12,49,50)$ have been suggested. In this sense, shorter data acquisition procedures and simplified monitoring tools to quantify data, such as the ultra-short-term HRV (HRV measured in $1 \mathrm{~min}$ post-1-min stabilization) were recently introduced to allow the detection of training adaptations in Futsal player (51). This suggests that ultra short-term HRV is sensitive to training effects in team sports players, thus of relevance in sport physiology.

In addition, the determination of HRV immediately post-exercise in different training regiment resulted in different changes in HRV. HF values during the first hour after exercise were higher after constant intensity training when compared with interval training. The early recovery of autonomic modulation from $5 \mathrm{~min}$ to $1 \mathrm{~h}$ after termination of exercise were greatly dependent on the allocation of training of exercise load. In particular, athletes showed a slower return of parasympathetic activity during short-term recovery after an interval (intensive) method of training relative to constant intensity of exercise (13). This suggests that the slower return may be due to withdrawal of the parasympathetic activity or for a more pronounced sympathetic involvement during the interval method relative to the stable method. By contrast, the late recovery of 24 and $48 \mathrm{~h}$ after termination of exercise did not depend on the type of exercise (interval training or constant identifying of exercise). Although it was concluded that the total exercise load (volume) determined long-term HRV recovery, it was found that trained athletes have different HRV post-exercise than less trained athletes (52). Changes could be identified at 2 min post-exercise recovery.
The use of HRV for monitoring and improving sports physiology. Optimal training depends on matching the specific ability of an athlete, such as muscle, strength, endurance, explosiveness, flexibility and adaptability to the individual's aerobic capacity, training load and recovery. For this purpose, the use of HRV is a suitable solution since it reflects the major regulatory processes after physical exercise. The use of HRV to detect which measures are altered versus physical exercise, type and intensity have been extended to demonstrate how monitoring physical fitness during exercise and post-exercise periods can be applied to athletic training more broadly in the future $(33,53)$. Long-term HRV changes during a prolonged period, over 4 weeks of exercise has been shown to be a particularly good indicator of physiological adaptation in athletes able to assist in the planning training programs. Previous findings showed that daily exercise intensity based on the HRV of the athlete, and lowering the intensity based on HRV decreased maintained fitness levels comparatively to the control groups $(54,55)$, indicating the importance of HRV use in sports physiology.

The usefulness of HRV measurements in prescribing exercise training in moderately active people have also been identified (56) within the prescription of standard training or HRV-guided training, including 2 months of moderate training $(70 \% \max \mathrm{HR})$ or vigorous training (85\% max HR). Additionally, the utility of HRV in daily endurance exercise prescription in 26 moderately fit males during a 4-week training period showed similarly beneficial outcomes in individuals who were prescribed lower-intensity exercise with decreased HRV, consistent with previous studies (57). However, these data were not reproducible in non-trained athletes or subjects with lower levels of physical ability $(2,55)$. Additionally, in athletes, HRV monitoring is frequently applied to prevent and diagnose overtraining (OT) syndrome (58), which is associated with numerous syndromes such as ANS dysfunction and imbalance (58). The test to diagnose fluctuations in ANS and the OT state $(33,59)$ is based on the measurement of the orthostatic HR that occurs between sitting and standing. Athletes in an OT state may show a significant decrease in frequency domain (TP, LF and HF) and time domain (RMSSDD and SDNN) variables. Additional observations (60) have yielded information revealing hyper-responsiveness in the frequency and time domain in OT athletes. Changes in HR do not occur in OT athletes with short-term training (i.e., 6 days) or long-term (6 months) OT $(58,61)$. Other findings have shown a predominance for LF (sympathetic) or HF (vagal) parameters $(61,62)$. This shift from vagal to sympathetic predominance has been reported in female athletes assayed for HRV in the supine rest position after a 6- to 9-week high-intensity training (63).

In junior cross-country skiers with reduced performance in competitions, early training breathlessness and central fatigue (64) were reported on the effects of OT. Power spectral analysis of the HRV at rest, during OT, and after recovery, showed that HF and TP were higher in the supine position compared to before and after OT, suggesting an extensive parasympathetic modulation during OT, in contrast to the sympathetic predominance identified during the 6 days of intensive boot camp received by these athletes and others in the OT state described in previous studies $(7,21,63)$. 
In the same registry, HRV change in 27 male and 30 female elite Nordic-skiers, examined between 2004 and 2008, were undertaken to characterize different types of 'fatigue'.

$\mathrm{R}-\mathrm{R}$ intervals recorded at rest during $8 \mathrm{~min}$ supine followed by $7 \mathrm{~min}$ standing for the analysis of LF, HF, LF+HF and HR, revealed that supine HRV patterns were independently sorted according to differently paired changes in the two postures. This characterization may be useful for further understanding of the autonomic rearrangement in different 'fatigue' conditions (21). HRV is used in the detection of the non-functional overreaching (NFOR) states, which are periods when athletic performance is substantially decreased, due to prolonged intensive training. Change in 34 elite female wrestlers ( 23 years old) were detected (60) using supine HRV analysis performed weekly at the same time of day, using the time and frequency domain before 11 competitions in the course of the study. Seven athletes were found in the NFOR state and 2 athletes in the FOR state (60).

The hypethesis behind the early detection of NFOR, OT and fatigue is the possibility of good recovery (35), involving the get of rest before training continues to allow repair to the body and to strengthen it between workouts. By allowing such a recovery to pre-training based on the constantly changing dynamic of the athlete and the amount of further training encountered or near pre-training levels, the recovery supports an optimizing future performance. The performance begins to decline if the recovery is not totally achieved as shown in the OT syndrome. In the OT syndrome, the training for an event or the event itself pushes beyond the body's ability to recover.

Thus, findings indicate the relevance of HRV measurements for the early identification of overreaching states and fatigues in professional athletes, which is of interest for sports physiology.

\section{Conclusion}

HRV is a non-invasive method used to obtain valuable data concerning physiological changes that occur in the response to physical activity. Findings resulting from multiple studies (7) suggest that HRV parameters are relevant in the analysis of stress that the body experiences during training and to increase insight into physiological recovery after training. Referring to athletes, changes in the patterns of their ANS reflected by altered HRV may serve as useful parameters for managing their physical fatigue and establishing their exercise intensity. Information regarding the extent to which the body recovers after training may provide useful data for the personalization of sports training, training loads and recovery times, targeting goal of improvement, avoiding OT and NFOR by keeping the athlete in a efficient frame work, which isimportant for sports physiology. However, standard protocols and methods for research with athletes should be established considering the intensity, the duration of exercise as well as the position of the body during recording and the duration of recording (14) for the use of HRV in sports physiology. Accordingly, the ultra-short-term HRV measurement method, demanding only 1 min of data acquisition after the stabilization period, may arguably improve the practicality of cardiac autonomic activity monitoring on a daily basis. This is relevant because the optimization of recovery requires the monitoring of HRV following workouts, which is crucial for the prevention of the extreme accumulation of physical fatigue during preparation or competition.

\section{References}

1. Boudoulas KD, Paraskevaidis IA, Boudoulas $H$ and Triposkiadis FK: The left atrium: From the research laboratory to the clinic. Cardiology 129: 1-17, 2014.

2. Rajendra Acharya U, Paul Joseph K, Kannathal N, Lim CM and Suri JS: Heart rate variability: A review. Med Biol Eng Comput 44: 1031-1051, 2006.

3. Hemingway H, Shipley M, Brunner E, Britton A, Malik M and Marmot M: Does autonomic function link social position to coronary risk? The Whitehall II study. Circulation 111: 3071-3077, 2005.

4. Boullosa DA, Tuimil JL, Leicht AS and Crespo-Salgado JJ: Parasympathetic modulation and running performance in distance runners. J Strength Cond Res 23: 626-631, 2009.

5. Khandoker AH, Jelinek HF and Palaniswami M: Identifying diabetic patients with cardiac autonomic neuropathy by heart rate complexity analysis. Biomed Eng Online 8: 3, 2009.

6. Tereshchenko LG, Cygankiewicz I, McNitt S, Vazquez R, Bayes-Genis A, Han L, Sur S, Couderc JP, Berger RD, de Luna AB, et al: Predictive value of beat-to-beat QT variability index across the continuum of left ventricular dysfunction: Competing risks of noncardiac or cardiovascular death and sudden or nonsudden cardiac death. Circ Arrhythm Electrophysiol 5: 719-727, 2012.

7. Amano M, Kanda T, Ue H and Moritani T: Exercise training and autonomic nervous system activity in obese individuals. Med Sci Sports Exerc 33: 1287-1291, 2001.

8. Oliveira RS, Leicht AS, Bishop D, Barbero-Álvarez JC and Nakamura FY: Seasonal changes in physical performance and heart rate variability in high level futsal players. Int J Sports Med 34: 424-430, 2013.

9. Plews DJ, Laursen PB, Kilding AE and Buchheit M: Heart rate variability in elite triathletes, is variation in variability the key to effective training? A case comparison. Eur J Appl Physiol 112: 3729-3741, 2012.

10. Kaikkonen P, Hynynen E, Mann T, Rusko H and Nummela A: Heart rate variability is related to training load variables in interval running exercises. Eur J Appl Physiol 112: 829-838, 2012.

11. Vesterinen V, Häkkinen K, Hynynen E, Mikkola J, Hokka L and Nummela A: Heart rate variability in prediction of individual adaptation to endurance training in recreational endurance runners. Scand J Med Sci Sports 23: 171-180, 2013.

12. Mourot L, Bouhaddi M, Tordi N, Rouillon JD and Regnard J: Short- and long-term effects of a single bout of exercise on heart rate variability: Comparison between constant and interval training exercises. Eur J Appl Physiol 92: 508-517, 2004.

13. Triposkiadis F, Karayannis G, Giamouzis G, Skoularigis J, Louridas $\mathrm{G}$ and Butler $\mathrm{J}$ : The sympathetic nervous system in heart failure physiology, pathophysiology, and clinical implications. J Am Coll Cardiol 54: 1747-1762, 2009.

14. Aubert AE, Seps B and Beckers F: Heart rate variability in athletes. Sports Med 33: 889-919, 2003.

15. Bosquet L, Papelier Y, Léger L and Legros P: Night heart rate variability during overtraining in male endurance athletes. J Sports Med Phys Fitness 43: 506-512, 2003.

16. Malpas SC and Purdie GL: Circadian variation of heart rate variability. Cardiovasc Res 24: 210-213, 1990.

17. Hill LK, Hu DD, Koenig J, Sollers JJ III, Kapuku G, Wang X, Snieder H and Thayer JF: Ethnic differences in resting heart rate variability: A systematic review and meta-analysis. Psychosom Med 77: 16-25, 2015.

18. Makivić B, Nikić M.D,Willis MS, Education P, \& Parovića, Heart rate variability (HRV) as a tool for diagnostic and monitoring performance in sport and physical activities. Volume 16 Number 3: 103-131, 2013.

19. Campbell GD, Edwards FR, Hirst GD and O'Shea JE: Effects of vagal stimulation and applied acetylcholine on pacemaker potentials in the guinea-pig heart. J Physiol 415: 57-68, 1989.

20. Ogliari G, Mahinrad S, Stott DJ, Jukema JW, Mooijaart SP, Macfarlane PW, Clark EN, Kearney PM, Westendorp RG, de Craen AJ, et al: Resting heart rate, heart rate variability and functional decline in old age. CMAJ 187: E442-E449, 2015. 
21. Schmitt L, Regnard J, Parmentier AL, Mauny F, Mourot L, Coulmy N and Millet GP: Typology of 'Fatigue' by Heart Rate Variability Analysis in Elite Nordic-skiers. Int J Sports Med 36: 999-1007, 2015.

22. Stein PK, Bosner MS, Kleiger RE and Conger BM: Heart rate variability: A measure of cardiac autonomic tone. Am Heart J 127: 1376-1381, 1994.

23. van Ravenswaaij-Arts CM, Kollée LA, Hopman JC, Stoelinga GB and van Geijn HP: Heart rate variability. Ann Intern Med 118: 436-447, 1993

24. Reed MJ, Robertson CE and Addison PS: Heart rate variability measurements and the prediction of ventricular arrhythmias. QJM 98: 87-95, 2005.

25. Aubert AE, Seps B and Beckers F: Heart rate variability in athletes. Sports Med 33: 889-919, 2003.

26. Mainardi LT, Bianchi AM, Baselli G and Cerutti S: Pole-tracking algorithms for the extraction of time-variant heart rate variability spectral parameters. IEEE Trans Biomed Eng 42: 250-259, 1995.

27. Pagani M, Lombardi F, Guzzetti S, Rimoldi O, Furlan R Pizzinelli P, Sandrone G, Malfatto G, Dell'Orto S and Piccaluga E: Power spectral analysis of heart rate and arterial pressure variabilities as a marker of sympatho-vagal interaction in man and conscious dog. Circ Res 59: 178-193, 1986.

28. Malik M and Camm AJ: Components of heart rate variability - what they really mean and what we really measure. Am J Cardiol 72: 821-822, 1993.

29. Tulppo MP, Mäkikallio TH, Takala TE, Seppänen T and Huikuri HV: Quantitative beat-to-beat analysis of heart rate dynamics during exercise. Am J Physiol 271: H244-H252, 1996.

30. Strano S, Lino S, Calcagnini G, Di Virgilio V, Ciardo R, Cerutti S Calcagnini $\mathrm{G}$ and Caselli G: Respiratory sinus arrhythmia and cardiovascular neural regulation in athletes. Med Sci Sports Exerc 30: 215-219, 1998.

31. de Oliveira Ottone V, de Castro Magalhães F, de Paula F, Avelar NC, Aguiar PF, da Matta Sampaio PF, Duarte TC, Costa KB, Araújo TL, Coimbra CC, et al: The effect of different water immersion temperatures on post-exercise parasympathetic reactivation. PLoS One 9: e113730, 2014.

32. Plews DJ, Laursen PB, Stanley J, Kilding AE and Buchheit M: Training adaptation and heart rate variability in elite endurance athletes: Opening the door to effective monitoring. Sports Med 43: 773-781, 2013

33. Hottenrott K, Hoos O and Esperer HD: Heart rate variability and physical exercise. Current status. Herz 31: 544-552, 2006 (In German).

34. Kaikkonen P, Rusko $\mathrm{H}$ and Martinmäki K: Post-exercise heart rate variability of endurance athletes after differen high-intensity exercise interventions. Scand J Med Sci Sports 18: 511-519, 2008

35. Kaikkonen P, Nummela A and Rusko $H$ : Heart rate variability dynamics during early recovery after different endurance exercises. Eur J Appl Physiol 102: 79-86, 2007.

36. Vanderlei LC, Silva RA, Pastre CM, Azevedo FM and Godoy MF: Comparison of the Polar S810i monitor and the ECG for the analysis of heart rate variability in the time and frequency domains. Braz J Med Biol Res 41: 854-859, 2008.

37. Lee S, Lee MS, Choi JY, Lee SW, Jeong SY and Ernst E: Acupuncture and heart rate variability: A systematic review. Auton Neurosci 155: 5-13, 2010

38. Posadzki P, Kuzdzal A, Lee MS and Ernst E: Yoga for Heart Rate Variability: A Systematic Review and Meta-analysis of Randomized Clinical Trials. Appl Psychophysiol Biofeedback 40: 239-249, 2015

39. Kemp AH, Quintana DS, Gray MA, Felmingham KL, Brown K and Gatt JM: Impact of depression and antidepressant treatment on heart rate variability: A review and meta-analysis. Biol Psychiatry 67: 1067-1074, 2010.

40. Cottin F, Médigue C, Leprêtre PM, Papelier Y, Koralsztein JP and Billat V: Heart rate variability during exercise performed below and above ventilatory threshold. Med Sci Sports Exerc 36 594-600, 2004.

41. Pichon AP, de Bisschop C, Roulaud M, Denjean A and Papelier Y: Spectral analysis of heart rate variability during exercise in trained subjects. Med Sci Sports Exerc 36: 1702-1708, 2004.

42. Chwyczko T, Sterliński M, Maciag A, Firek B, Labecka A Jankowska A, Kośmicki M, Kowalik I, Malczewska B and Szwed H: Impact of cardiac resynchronisation therapy on adaptation of circulatory and respiratory systems to exercise assessed by cardiopulmonary exercise test in patients with chronic heart failure. Kardiol Pol 66: 406-412; discussion 413-414, 2008
43. He X, Zhao M, Bi X, Sun L, Yu X, Zhao M and Zang W: Novel strategies and underlying protective mechanisms of modulation of vagal activity in cardiovascular diseases. Br J Pharmacol 172: 5489-5500, 2015

44. Tulppo MP, Mäkikallio TH, Seppänen T, Laukkanen RT and Huikuri HV: Vagal modulation of heart rate during exercise: Effects of age and physical fitness. Am J Physiol 274: H424-H429, 1998.

45. Berkoff DJ, Cairns CB, Sanchez LD and Moorman CT III: Heart rate variability in elite American track-and-field athletes. J Strength Cond Res 21: 227-231, 2007.

46. Hautala A, Tulppo MP, Mäkikallio TH, Laukkanen R, Nissilä S and Huikuri HV: Changes in cardiac autonomic regulation after prolonged maximal exercise. Clin Physiol 21: 238-245, 2001.

47. de Freitas VH, Pereira LA, de Souza EA, Leicht AS, Bertollo M and Nakamura FY: Sensitivity of the Yo-Yo Intermittent Recovery Test and cardiac autonomic responses to training in futsal players. Int J Sports Physiol Perform 10: 553-558, 2015.

48. Soares-Caldeira LF, de Souza EA, de Freitas VH, de Moraes SM, Leicht AS and Nakamura FY: Effects of additional repeated sprint training during preseason on performance, heart rate variability, and stress symptoms in futsal players: A randomized controlled trial. J Strength Cond Res 28: 2815-2826, 2014.

49. Flatt AA and Esco MR: Smartphone-Derived Heart-Rate Variability and Training Load in a Women's Soccer Team. Int J Sports Physiol Perform 10: 994-1000, 2015.

50. Plews DJ, Laursen PB, Le Meur Y, Hausswirth C, Kilding AE and Buchheit $\mathrm{M}$ : Monitoring training with heart rate-variability: How much compliance is needed for valid assessment? Int J Sports Physiol Perform 9: 783-790, 2014.

51. Nakamura FY, Flatt AA, Pereira LA, Ramirez-Campillo R, Loturco I and Esco MR: Ultra-Short-Term Heart Rate Variability is Sensitive to Training Effects in Team Sports Players. J Sports Sci Med 14: 602-605, 2015

52. Seiler S, Haugen O and Kuffel E: Autonomic recovery after exercise in trained athletes: Intensity and duration effects. Med Sci Sports Exerc 39: 1366-1373, 2007.

53. Buchheit M, Millet GP, Parisy A, Pourchez S, Laursen PB and Ahmaidi S: Supramaximal training and postexercise parasympathetic reactivation in adolescents. Med Sci Sports Exerc 40: 362-371, 2008

54. Pichot V, Busso T, Roche F, Garet M, Costes F, Duverney D, Lacour JR and Barthélémy JC: Autonomic adaptations to intensive and overload training periods: A laboratory study. Med Sci Sports Exerc 34: 1660-1666, 2002

55. Pichot V, Roche F, Gaspoz JM, Enjolras F, Antoniadis A, Minini P, Costes F, Busso T, Lacour JR and Barthélémy JC: Relation between heart rate variability and training load in middle-distance runners. Med Sci Sports Exerc 32: 1729-1736, 2000.

56. Kiviniemi AM, Hautala AJ, Kinnunen H, Nissilä J, Virtanen P, Karjalainen J and Tulppo MP: Daily exercise prescription on the basis of HR variability among men and women. Med Sci Sports Exerc 42: 1355-1363, 2010.

57. Kiviniemi AM, Hautala AJ, Kinnunen $\mathrm{H}$ and Tulppo MP: Endurance training guided individually by daily heart rate variability measurements. Eur J Appl Physiol 101: 743-751, 2007.

58. Mourot L, Bouhaddi M, Perrey S, Cappelle S, Henriet MT, Wolf JP, Rouillon JD and Regnard J: Decrease in heart rate variability with overtraining: Assessment by the Poincaré plot analysis. Clin Physiol Funct Imaging 24: 10-18, 2004

59. Hynynen E, Uusitalo A, Konttinen N and Rusko H: Heart rate variability during night sleep and after awakening in overtrained athletes. Med Sci Sports Exerc 38: 313-317, 2006.

60. Tian Y, He ZH, Zhao JX, Tao DL, Xu KY, Earnest CP and McNaughton LR: Heart rate variability threshold values for early-warning nonfunctional overreaching in elite female wrestlers. J Strength Cond Res 27: 1511-1519, 2013.

61. Hedelin R, Kenttä G, Wiklund U, Bjerle $P$ and Henriksson-Larsén K: Short-term overtraining: Effects on performance, circulatory responses, and heart rate variability. Med Sci Sports Exerc 32: 1480-1484, 2000.

62. Hedelin R, Wiklund U, Bjerle P and Henriksson-Larsén K: Cardiac autonomic imbalance in an overtrained athlete. Med Sci Sports Exerc 32: 1531-1533, 2000.

63. Uusitalo AL, Uusitalo AJ and Rusko HK: Heart rate and blood pressure variability during heavy training and overtraining in the female athlete. Int J Sports Med 21: 45-53, 2000

64. Quintana DS, Heathers JA and Kemp AH: On the validity of using the Polar RS800 heart rate monitor for heart rate variability research. Eur J Appl Physiol 112: 4179-4180, 2012. 